OnLine Journal of Biological Sciences 11 (1): 23-26, 2011

ISSN 1608-4217

(C) 2011 Science Publications

\title{
Entropyomics as the Blueprint of the Logic of Normal Cell Division and Malignancy
}

\author{
Kambiz Afrasiabi \\ Department of Medicine and Pathology, \\ University of California, Irvine, California, USA
}

\begin{abstract}
Problem statement: In this article I propose a blueprint based on one of the most fundamental laws governing the known universe, namely the second law of thermodynamics and I present support for its central role in initiation of mitosis and relationship of the other sub cellular compartments and their organization. Approach: Life is considered to be the most sophisticated antientropy machinery ever born on the face of the universe as far as its power to minimize the speed of rise in entropy is concerned, however we all get old, sick and die because it is not possible to stop the rise in entropy based on the nature of the known universe. Results: Lack of understanding of the scientific foundation of logic of the normal cell division has surrounded us by darkness and has made analysis of an ever increasing and explosive amount of information originating from whole genome sequencing, genomics, exonomics, proteomics and metabolomics more problematic. Clearly this understanding is the prerequisite for understanding of pathological states of cell division including malignancy. Conclusion/Recommendations: The main approach to this problem is calculation of the free energy of the master regulator proteins of the intracellular communication network of the cancer stem cell and its normal counterpart which in turn could get identified by the available mathematical models that could identify master regulator proteins of the intracellular communication network and deciphering the difference by spectrophotometry at a given wavelength of light and identification of higher absorbance in the malignant counterpart and designing epigenetic or homologous recombination mediated methodology using nanotechology as a delivery mechanism targeting transcription of mRNAs which would lead to protein products with a normal free energy for that cell lineage / higher free energy compared with its malignant counterpart and by doing so we could convert the cancer stem cell pool to a normal one, i.e.: achieve cure.
\end{abstract}

Key words: Stem cell, anti-entropy machinery, cell energetics, cell division, homologous recombination, transcribed RNA, microRNA, cancer therapeutics, master regulator, protein products, entropy and cancer, entropy

\section{INTRODUCTION}

At the time of fertilization entropy of the fertilized ovum increases dramatically and instantaneously as a result of increase in the number of chromosomes in the same amount of space, i.e., Crowding which correlates with a decrease in free energy, in addition the difference between the maternal and paternal imprinting signatures which increases the number of variables contributes to increase in entropy. I propose that this sudden decrease in free energy and increase in entropy acts as a shock wave to the fabric of the bio-unit which is inherently an anti entropy machinery and that triggers mitosis as an attempt to reverse the process and because the total number of chromosomes remains the same in each cell, compensation happens by increase in the free energy of the constituents of the cell and throughout embryogenesis the free energy of the genome through epigenetic modifications and that of the RNA and protein products of the future generation cells would incrementally continue to increase with each subsequent round of mitosis until it reaches the limit of function of $\mathrm{x}$ and this could coincide with a progressive decrease in the difference between the maternal and paternal imprinting signature through this convoluted pathway of recurrent rounds of mitosis that I have referred to in my previous publication entitled quantum stem cell in the infinity loop embryogenesis which is not necessarily meant to be the main goal of this pathway would happen. We can now start to address the many different riddles of the various cellular sub compartments of the cell and functional 
intracellular network by applying the general principles that govern this blue print. The proof of this blue print demands single cell profiling of the energetics of the zygote and all the ensuing cells throughout embryogenesis, i.e., measurement of free energy of chromatin, RNA and proteins of each cell generation or a representative sample of them in these cells based on mathematical models defining master regulators; however cumbersome this task might seem it is the only way to the understanding of the normal state and description of its abnormalities and its completion would lead to creation of the most fundamental road map in biology which is missing today and without which we will continue to define the elephant in the dark room in new ways every day. By the same token all the sub-cellular compartments are expected to cooperate for the achievement of the same goal, namely increase in free energy and decrease in entropy and any perturbation that leads to a decrease in free energy or inappropriate increase in entropy of the stem cell compartment of any given organ would activate another round of mitosis to restore the baseline free energy level of that compartment. These perturbations could originate in the environment or happen as a result of ill mutations or a combination of both. Lack of ability to correct these abnormalities on behalf of the cell would lead to over population of that compartment with cells which have an inappropriately low free energy/high entropy, thereafter the cell starts to employ different mechanisms to overcome this hurdle a few example of which are over-expression of kinases to increase the pace of mitosis and certain mutations to prevent apoptosis which would give the cell more time to repair the defect and in the case of a full blown malignancy this becomes an impossible task to achieve. Through understanding and dissection of the concept of this blueprint we could correct so many false definitions and misunderstandings and potentially cure malignancy. Clearly the final cure of malignancy demands modification/ increase of the free energy of the stem cell compartment of the malignant organ to its baseline normal level through the design of new epigenetic mediated treatment modalities which would lead to the generation of RNAs and microRNAs which would lead to formation of protein products with the desired free energy; other sub compartments of the cell could also get employed along the same line, e.g., Proteosome inhibitors to minimize the destruction of the desired protein products or activation of homologous recombination machinery in a way that the new DNA product would fulfill the pre set goals and parameters. This is in need of a lot of sophisticated technology and delivery system and nano technology seems to be an appropriate delivery modality; by the same token the final cure of cancer denies destruction which is the most common treatment modality nowadays, namely chemo and radiation therapy. Even our targeted therapies of today such as kinase inhibitors and monoclonal antibodies are directed towards the branches of a deeply seated root. I would like to address several major examples of the shortcomings in our current understanding of the different sub-cellular functions and their potential therapeutic applications (Afrasiabi, 2010; Calin, 2006; Scaffidi and Misteli, 2010; Tong et al., 2010).

Homologous recombination which is one of the main machineries for repair of double strand DNA break, opposite to what we currently think could happen either following internal homeostatic processes guided by the master regulator mechanisms or under external environmental factors, i.e., A-Either following self induced or b-Exogenous agent (s) induced double strand DNA break. The transcribed RNA and translated protein product of the repaired master regulator induced double strand DNA break is expected to have the highest possible free energy or the lowest entropy. In other words there is constant monitoring and vigilance of the free energy status of the chromatin and the transcribed RNA and translated proteins and a constant drive towards the lowest entropy or highest free energy protein products by master regulator network; along the same line the complex microRNA network and histone modifications would be serving the same simple purpose and are constantly under scrutiny by master regulator network. We could potentially design double strand DNA breaks by using nano technology nucleoside sequence specific directed approach to generate genes or epigenes the protein products of which would have the highest free energy and the lowest entropy and replace the pathological master regulator proteins which have inappropriately low free energy and high entropy compared with their normal tissue counterparts with these nano products, the same methodology could get applied to modify histone code and micro RNA network.

Exons and introns: We all have learned that exons are that part of the genome which code RNA for the purposeful formation of useful biological proteins and introns occupy the space between the exons. Most recently we have also learned that microRNAs could get coded by the introns. It is expected that as we get 
older the free energy of the epigenome, RNA and protein products of any well defined exon would decrease as a result of escape from corrective mechanisms and this is an obligation dictated by the second law of thermodynamics, in other words the old exon of any known gene is expected to have got modified to a less fit one as far as free energy of product is concerned; through this blue print master regulator network's constant vigilance of the epigenome, subtle rearrangements of the exon which could incorporate interactions with microRNAs, post transcriptional modifiers and RNA splicing machinery would aim at maximizing the free energy of protein products of the genome. Thus as we get older, i.e., as our free energy decreases RNA splicing and poly adenylation of the same RNA happens at a different site to satisfy the same result because the older exon has changed to one with a lower free energy. By the same token post translational modifications would be following the same theme as escape from these corrective mechanisms and pathways is eventually inevitable and epigenomes, RNA and protein products with lower free energy and higher entropy would dominate, take over and lead the system towards a vast array of disease processes including cancer, it is also expected that the proteo some machinery whose major job is to get rid of low free energy/unfit proteins would itself get affected by the same aging process and along the same line telomeres which are the biological clocks monitoring the total number of the allowable rounds of mitosis before catastrophic genetic events prevail do not get shorter in the vast majority of cancers and the unfit cell does not undergo apoptosis and incessant rounds of mitosis would lead to overpopulation of the organ with such ill cells and employing further mutations and translocations by the stem cell compartment which is comprised of around one percent of cells in each organ in which free energy has reached the maximum possible level during embryogenesis and I would prefer to call them organ creator cells would add to the depth of catastrophe as the system has already escaped to infinity, i.e.: Entropy increase has escaped maximum capture capability and no finite measure would be able to handle the problem. Cancer therapeutic strategies aiming at shortening telomeres ignore the fact that the new generation of cells replacing the ones that die following shortening of telomere would still have epigenomes that generate low free energy dysfunctional proteins and enzymes that lead to formation of long telomere and long telomere in cancer cells is an effect and not a cause of malignancy. At the end 1 would like to touch on one disease example, namely prostate cancer which is the most common cancer affecting men in the USA and the proposed treatment strategy using this blueprint; we have learned that the major difference between a prostate cancer cell and its normal counterpart is in the increased number of androgen receptors/AR's in the nucleus of the prostate cancer cell compared with the normal cell. The weight of evidence suggests that the complex network of the chaperone and transporter proteins becomes defective leading to increased trafficking of AR's and their spliced variants into the nucleus causing castrate resistance. One potential explanation for the increased trafficking of AR's into the nucleus is the loosening of the tight association between these chaperone proteins and the AR's in the cytoplasm as a result of change in their stoichiometry following a decrease in their free energy/increase in their entropy which could happen following a diverse group of possibilities ranging from alteration in the epigenetic to micro RNA's or aberrant splicing secondary to escape from corrective mechanisms as a complication of ill exogenous and/or endogenous agents including mutations, oxidative damage or viral infections. In order to cure prostate cancer we must become able to calculate the free energy of the master regulator(s) of this network and decipher the difference with their normal counterpart and then design a corrective epigenetics or micro RNA based or post translational modification strategy restoring the free energy of the master regulator chaperone proteins to their baseline/normal level; sequence specific targeted homologous recombination could also be used to achieve the same end result, we can now imagine how far we are from those days and how crude our current treatment modalities are.

$\mathrm{X}$ Ray crystallography and nano imaging of the master regulator complex at each given stage of embryogenesis as well as the cancer stem cell compartment target complex of interest and calculating the absorbance of the emitted photon beam on real time structure of the cancer target complex and its normal counterpart at a predetermined wavelenght would determine the free energy of each complex.

The absorbance of light by the target master regulator complex of cancer stem cell compartment is significantly higher than its normal counterpart which correlates with its higher entropy.

We are in need of new and revolutionary approach to the old problem of malignancy and its cure. Our current approach is rudimentary and primitive given the depth, scientific reality and the roots of the problem and is ignorant of the facts govrning the normal state 
and malignancy, we should move fast and redirect our treatment strategies based on the differences in the energetics of normal cell and its malignant counterpart and by using new imaging and delivery mechanisms incorporating nano technology land our conversion curative modality by using epigenetics and micro RNA as targets and by approaches such as sequence specific homologous recombination pursue that goal; we should also seek a new source of research funding away from the quick gratificaion pharmaceutical derived machinary.

\section{CONCLUSION}

The conscious cell has a conscious mind and is born in a conscious universe which obeys certain sets of universal laws with a central role for the second law of thermodynamics. The biouniverse is a masterpiece in which the speed of rise of entropy is the slowest in comparison with all the other systems in the whole known universe. Aberrancies in the homeostasis of this law could lead to catastrophic events including malignancy and it is only when we come to understand the fine regulation of energetics of the cell that we could become able to understand and cure cancer. We need to rush in mapping the energetics of the normal and disease states and by using the finest delivery methodologies such as nano technology reinstate the normal energetics of the cell in a given tissue and only then could we achieve cure of catastrophic diseases such as cancer.

\section{REFERENCES}

Afrasiabi, K., 2010. Quantum stem cell in the infinity ioop. Online J. Biol. Sci., 10: 60-65. DOI: 10.3844/ojbsci.2010.60.65

Calin, G.A., 2006. Micro RNA signatures in human cancers. Nature Rev. Cancer, 6: 857-866. DOI: 10.1038/nrc1997

Scaffidi, P. and T. Misteli, 2010. Cancer epigenetics: From disruption of differentiation programs to the emergence of cancer stem cells. Cold Spring Harb. Symp. Quant. Biol. DOI: 10.1101/sqb.2010.75.007

Tong, C., P. Li, N.L. Wu, Y. Yan and Q.-L. Ying, 2010. Production of p53 gene knockout rats by homologous recombination in embryonic stem cells. Nature Lett., 467: 211-213. DOI: 10.1038/nature09368 